\title{
Comparison of erector spinae plane block and paravertebral block for breast surgery: a retrospective analysis
}

Ritsuko Hirade $^{1}$, Shinichi Sakura ${ }^{1}$, Aumjit Wittayapairoj ${ }^{2}$, Yuki Aoyama ${ }^{1}$, Yoji Saito ${ }^{1}$

1. Shimane University Hospital, Department of Anesthesiology, Izumo, Japan

2. Department of anesthesiology, Faculty of Medicine, Khon Kaen University, Khon Kaen, Thailand

\section{Background and Aims}

$\checkmark$ Thoracic paravertebral block (PVB) is an established technique for perioperative pain management for breast surgery.

$\checkmark$ Since PVB is technically challenging, it has not been widely used.

$\checkmark$ Recently introduced erector spinae plane block (ESPB) requires less technical expertise and may be an alternative to PVB.

$\checkmark$ The two blocks have not been fully compared.

$\checkmark$ The present study retrospectively analyzed data saved in our registry to compare the two blocks in patients undergoing breast surgery.

\section{Methods}

$\checkmark$ A retrospective study using regional anesthesia database in our department

$\checkmark$ The data of patients undergoing breast surgery who received either PVB or ESPB under ultrasound guidance from June 2018 to June 2019
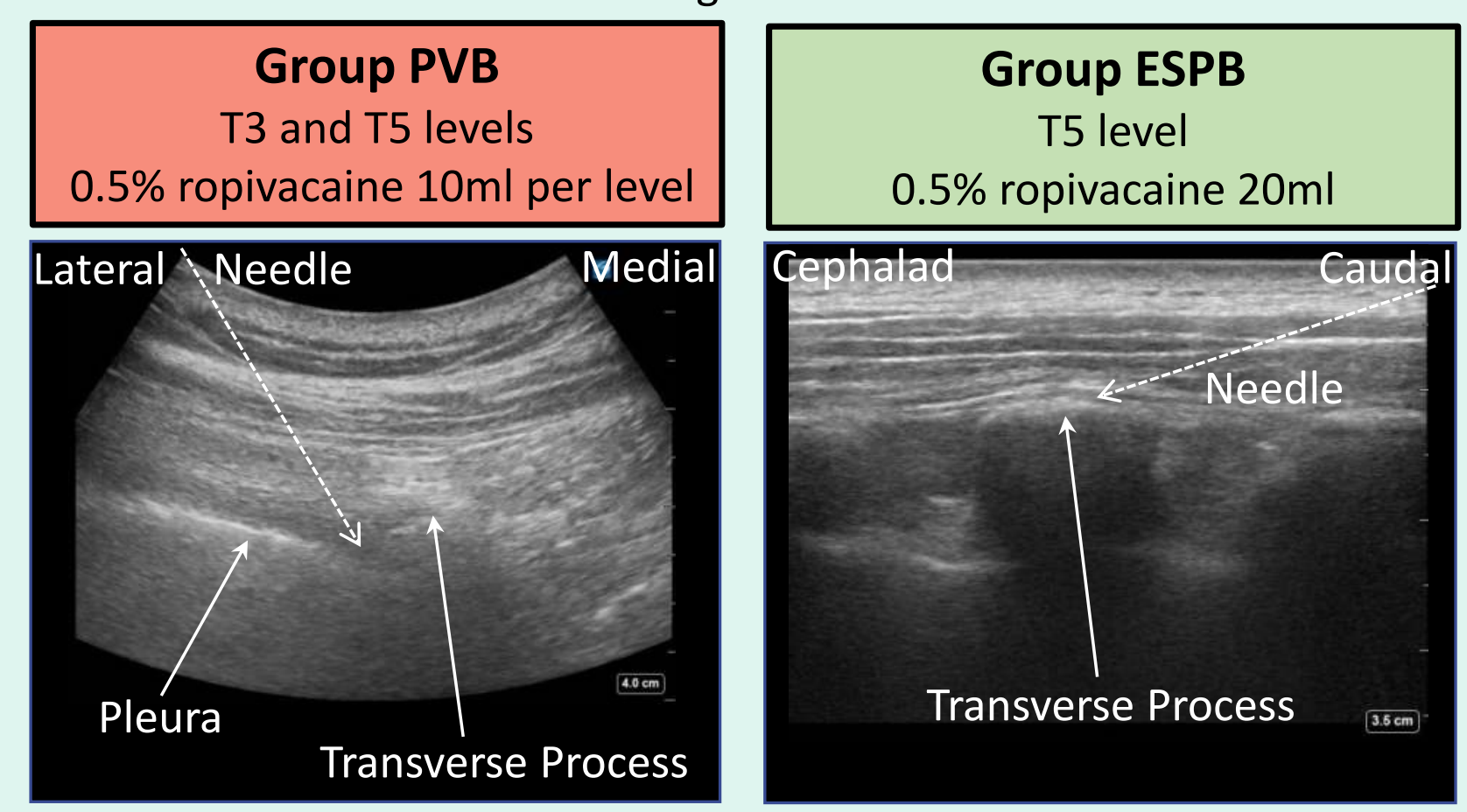

\section{Measurements}

$\checkmark$ The primary outcome: VAS pain scores at $12 \mathrm{~h}$ after block

$\checkmark$ The secondary outcomes:

- Scanning time, block performing time

- Presence of sensory blockade

- VAS pain scores for $24 \mathrm{~h}$

- Postoperative use of fentanyl from PCA device

"No basal infusion with $0.5 \mathrm{mcg} / \mathrm{kg}$ on demand bolus $/ 10$ minutes lockout

$(\mathrm{mm})$$$
\begin{array}{r}
100 \\
80 \\
60 \\
40 \\
20 \\
0
\end{array}
$$

\section{VAS at rest}

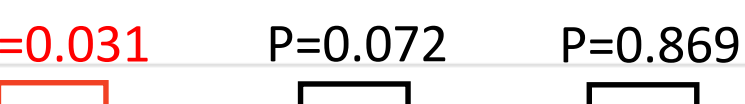

80 PVB

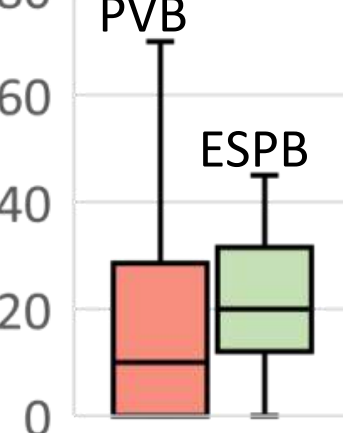

6

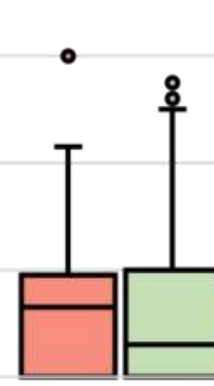

24
Time after block $(\mathrm{h})$

Loss of cold sensation 15min after block

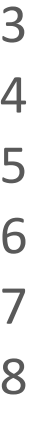

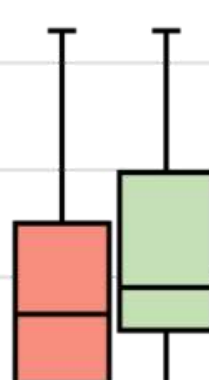

12
$P=0.869$

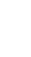

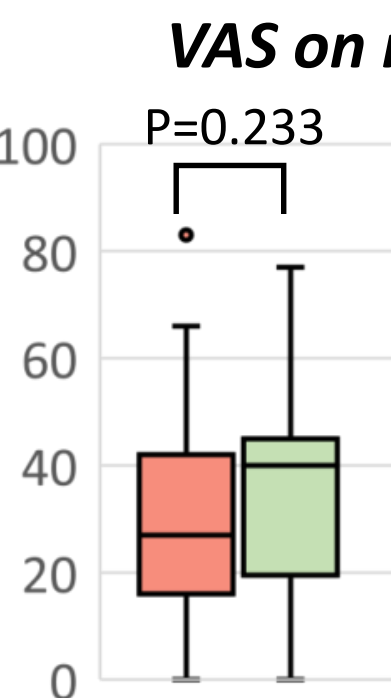

6

Time after block $(\mathrm{h})$

Loss of cold sensation at $6 \mathrm{~h}$

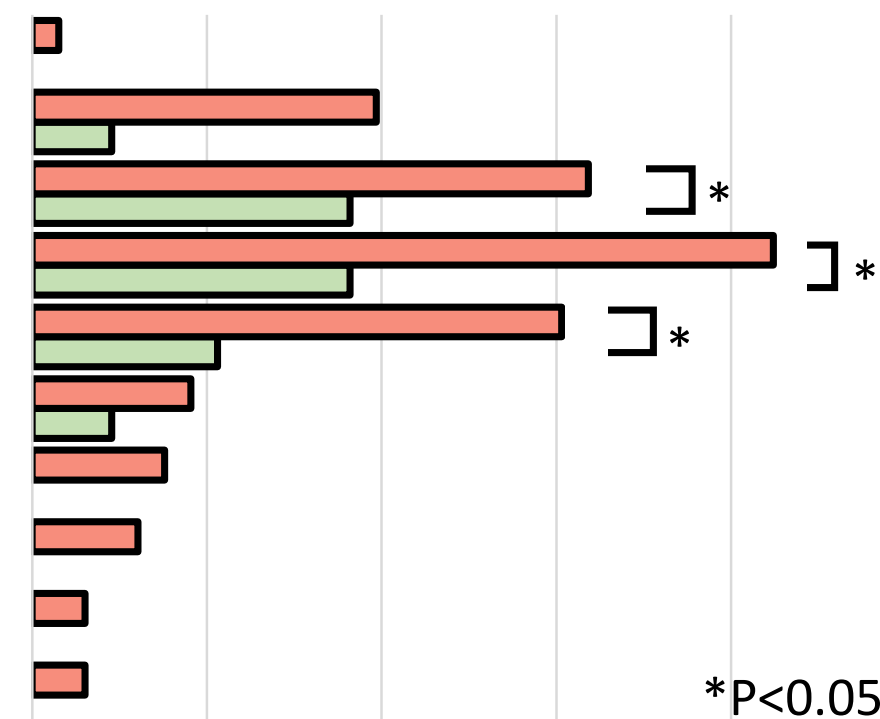

Results

Patient flow diagram

Patients undergoing breast cancer surgery ( $n=98)$

Patients given PNBs $(\mathrm{n}=71)$

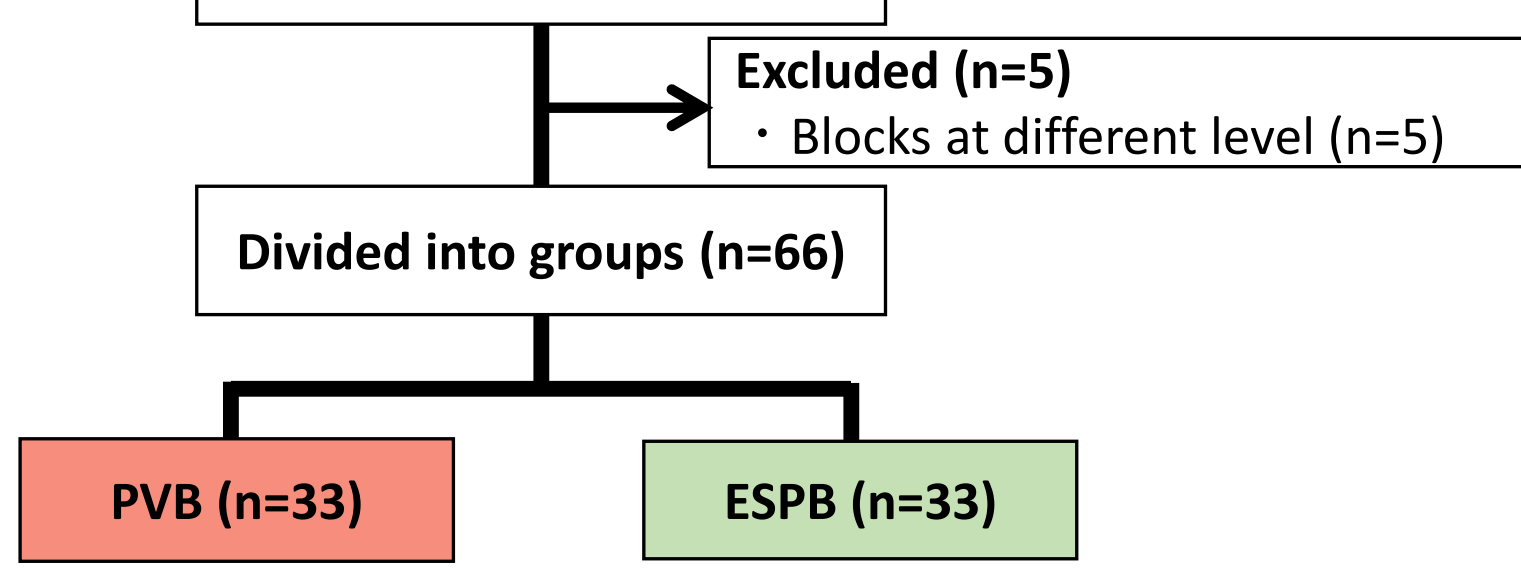

Demographics

\begin{tabular}{lccc} 
& PVB (n=33) & ESPB (n=33) & P-value \\
\hline Age(yr) & $56 \pm 17$ & $60 \pm 13$ & 0.245 \\
Body Weight (kg) & $54.1 \pm 9.7$ & $53.5 \pm 11.5$ & 0.800 \\
Height (cm) & $156.4 \pm 8.3$ & $154.1 \pm 5.8$ & 0.191 \\
Gender, male/female & $1 / 32$ & $1 / 32$ & 1.000 \\
ASA PS, I/II/III & $8 / 25 / 0$ & $5 / 26 / 2$ & 0.258 \\
Op Side, right/left & $15 / 18$ & $12 / 21$ & 0.617 \\
Type of surgery: & & & 0.687 \\
- Bp + SLNB & 10 & 13 & \\
- Bt + SLNB & 14 & 11 & \\
- Bp or Bt + node clearance & 9 & 9 & \\
Surgical time (min) & $136 \pm 55$ & $151 \pm 60$ & 0.278 \\
Anesthetic time (min) & $197 \pm 56$ & $213 \pm 67$ & 0.279 \\
Intraoperative opioids used: & & & \\
\hline - fentanyl (mcg) & $247 \pm 91$ & $227 \pm 63$ & 0.276 \\
- remifentanil (mcg) & $794 \pm 440$ & $1030 \pm 558$ & 0.061
\end{tabular}

Data were expressed as mean \pm SD or number of patients. $\mathrm{Bp}=$ partial resection. $\mathrm{Bt}=$ total resection. SLNB = sentinel lymph node biopsy.

\section{Scanning and performing time}

\begin{tabular}{lccc} 
& PVB $(n=33)$ & ESPB $(n=33)$ & P-value \\
\hline Scanning time (sec) & $171 \pm 89$ & $113 \pm 89$ & 0.011 \\
\hline Block performing time (sec) & $369 \pm 180$ & $190 \pm 97$ & $<0.001$ \\
\hline
\end{tabular}

\section{Analgesic requirements and adverse events}

\begin{tabular}{lccc}
\hline & PVB $(n=33)$ & ESPB $(n=33)$ & P-value \\
\hline $\begin{array}{l}\text { Postoperative fentanyl } \\
\text { consumption }(\mathrm{mcg})\end{array}$ & $27(0-75)$ & $47(0-106)$ & 0.279 \\
Nausea & $7(21 \%)$ & $8(24 \%)$ & 1.000 \\
Vomiting & $5(15 \%)$ & $2(6 \%)$ & 0.427 \\
\hline
\end{tabular}

Data were expressed as median (IQR) or number of patients (\%).

Presence of sensory blockade

\begin{tabular}{lccc}
\hline & PVB(n=33) & ESPB $(n=33)$ & $P$ value \\
\hline 15 min after block(n(\%)) & $32(97 \%)$ & $7(21 \%)$ & $<0.001$ \\
6 hours after block(n(\%)) & $31(94 \%)$ & $17(52 \%)$ & $<0.001$
\end{tabular}

\section{Conclusion}

$\checkmark$ The two blocks had different characteristics, but were similar in pain scores at $12 \mathrm{~h}$ after block. 